\title{
Questes
}

vestes Revue pluridisciplinaire d'études médiévales

$26 \mid 2013$

Trouver la paix

\section{Trouver la paix : avant-propos}

\section{Gisela Naegle}

\section{(2) OpenEdition}

\section{Journals}

Édition électronique

URL : http://journals.openedition.org/questes/1335

DOI : 10.4000/questes.1335

ISSN : 2109-9472

\section{Éditeur}

Les Amis de Questes

\section{Édition imprimée}

Date de publication : 25 septembre 2013

Pagination : 3-8

ISSN : 2102-7188

\section{Référence électronique}

Gisela Naegle, «Trouver la paix : avant-propos », Questes [En ligne], 26 | 2013, mis en ligne le 01 janvier 2014, consulté le 23 septembre 2020. URL : http://journals.openedition.org/questes/1335; DOI : https://doi.org/10.4000/questes.1335 


\section{Avant-propos}

\section{Gisela NAEGLE}

Docteur en histoire de l'université de Gießen (Allemagne)

«Trouver la paix » : l'intitulé choisi pour ce numéro de Questes est un beau titre. Depuis des siècles, « trouver la paix » est un souhait universel qui se reflète également dans des formules comme "pax vobiscum» ou dans la définition de saint Augustin que Stéphanie Richard et Irène Strobbe ont choisie comme point de départ de leur introduction ${ }^{1}$. Cette définition souligne la tranquillitas ordinis qui va de la paix intérieure de l'individu jusqu'à celle du monde entier. Des idées semblables et d'autres tentatives de définir la paix se retrouvent dans les œuvres de nombreux auteurs médiévaux, par exemple dans le De bono pacis du canoniste Rufinus (vers 1180-1182) de l'université de Bologne. Pour lui, il y a trois «types » de paix qui n'ont pas la même qualité : la Pax Aegypti est une conspiration criminelle du diable, qui, finalement, mène au contraire de la paix (« in unam pravitatem conspiratio »); la Pax Babyloniae est la paix dans le monde ("ab externo vel civili bello privatave rixa tuta conversatio ») et la Pax Jerusalem est la paix chrétienne («christiane societatis fraternitas ») 2 . Cette classification est complétée par quatre catégories supplémentaires de paix : «pax orbis », «pax urbis », «pax domus» (avec les parents et les serviteurs) et amicitia (la paix fondée sur l'amitié) ${ }^{3}$.

\footnotetext{
${ }^{1}$ Voir l'introduction de Stéphanie Richard et Irène Strobbe, infra, p. 9-18.

2 Rufinus von Sorrent, De bono pacis, éd. Roman Deutinger, Hannover, Hahn, 1997, p. 102-103.

Rufinus, De bono pacis, éd. cit., « De tribus vel quatuor gradibus pacis mundane», p. 134-137.
} 
La paix est un sujet qui ne perdra jamais son actualité, un état auquel on aspire, mais qui est toujours fragile et en danger. Au Moyen Âge, comme aux époques ultérieures, il y eut des projets pour parvenir à une paix mondiale et durable ${ }^{4}$ et des mouvements de paix, dont celui pour la « paix de Dieu ». ${ }^{5}$ Mais la paix était également liée à la guerre, et pour des auteurs de projets de croisade comme Pierre Dubois ( $†$ après 1321) et les papes, la paix entre les princes chrétiens était un préalable nécessaire au lancement d'une croisade ${ }^{6}$. Selon un passage du Décret, la guerre pouvait être un moyen pour parvenir à la paix : «Non pax queritur, ut bellum exerceatur, sed bellum geritur, ut pax acquiratur ${ }^{7}$. En disant «Qui desiderat pacem, praeparat bellum ${ }^{8}$, Végèce, un auteur qui fut beaucoup lu et cité au Moyen Âge, notamment par Jean Juvénal des Ursins et Enea Silvio Piccolomini (le futur Pie II), allait encore plus loin ${ }^{9}$.

Au cours des dernières décennies, la paix et ses différents aspects ont fait l'objet de nombreuses publications dans divers pays européens;

${ }^{4}$ Pierre Monnet, «Le projet de paix et d'union chrétiennes de Georges de Podiebrad en 1462-1464 », dans Histoire du monde au $X V^{e}$ siècle, dir. Patrick Boucheron, Pierre Monnet et al., Paris, Fayard, 2009, p. 527-533 ; Lotte Kéry, «Pierre Dubois und der Völkerbund. Ein >Weltfriedensplan〈 um 1300 », Historische Zeitschrift, 283, 2006, p. 1-30 ; Busso Diekamp, Ewiger Landfrieden, 1495, Zum ewigen Frieden, 1795 : die Friedensidee vom Mittelalter bis zu Immanuel Kants philosophischem Entwurf «Zum ewigen Frieden», Worms, Stadtbibliothek, 1995.

5 Dominique Barthélemy, L'an mil et la paix de Dieu : la France chrétienne et féodale 980-1060, Paris, Fayard, 1999 ; Thomas Gergen, Pratique juridique de la paix et trêve de Dieu à partir du concile de Charroux (989-1250), Frankfurt/Main, Lang, 2004 ; Mihai-D. Grigore, Ehre und Gesellschaft. Ehrkonstrukte und soziale Ordnungsvorstellungen am Beispiel der Gottesfrieden (10-11. Jahrhundert), Darmstadt, WBG, 2009 ; Klaus Arnold, Mittelalterliche Volksbewegungen für den Frieden, Stuttgart, Kohlhammer, 1996.

${ }^{6}$ Jacques Paviot, "Faire la paix pour faire la guerre. Paix et guerre dans la croisade », dans Frieden schaffen und sich verteidigen im Spätmittelalter / Faire la paix et se défendre à la fin du Moyen Âge, dir. Gisela Naegle, Munich, Oldenbourg, 2012, p. 315-327.

Decreti Secunda Pars, Causa XIII, Quest. 1, c. 3 (Corpus Iuris Canonici, éd. Emil Friedberg, Leipzig, Tauchnitz, 1879, t. 1, col. 892).

${ }^{8}$ Vegetius, De re militari, 3 Prologus, dans Epitoma rei militaris, éd. Alf Önnerfors, Stuttgart, Leipzig, Teubner, 1995, p. 101.

${ }^{9}$ Gisela Naegle, «"Qui desiderat pacem, preparat bellum” : Guerre et paix chez Jean Juvénal des Ursins et Enea Silvio Piccolomini », dans Frieden schaffen / Faire la paix..., op. cit., p. 267-314. 
cependant, à cause de barrières linguistiques, les études comparées ne sont pas encore très fréquentes ${ }^{10}$. Les différentes approches retenues se reflètent déjà dans les titres qui se réfèrent à la prédication ${ }^{11}$, aux idées de paix, ${ }^{12}$ à ses « instruments » et acteurs ${ }^{13}$, à ses paroles ${ }^{14}$ et rituels ${ }^{15}$, aux discours et gestes $^{16}$, à l'action de faire la paix et aux nécessités de la défense ${ }^{17}$, ou, comme le $136^{\mathrm{e}}$ Congrès des sociétés historiques et scientifiques de Perpignan (2011), à la tension entre la paix et la guerre ${ }^{18}$. La paix donne lieu à des négociations diplomatiques et des traités solennels ${ }^{19}$, y parvenir

${ }^{10}$ Pour la deuxième moitié $\mathrm{du} \mathrm{xx}^{\mathrm{e}}$ siècle, voir par exemple Recueils de la Société Jean Bodin, La Paix, 2 vol., Éditions de la Librairie encyclopédique, Bruxelles, 1958-1962; La pace nel pensiero, nella politica, negli ideali del Trecento, Convegni del Centro di Studi sulla spiritualità medievale, 15, Todi, Accademia tudertina, 1975 ; Thomas Renna, « The Idea of Peace in the West, 500-1150 », Journal of Medieval History, 6, 1980, p. 143-167. Pour un aperçu bibliographique récent voir Gisela Naegle, «Bibliographie sélective » dans Frieden schaffen / Faire la paix..., op. cit., p. 383-400.

${ }^{11}$ Prêcher la paix et discipliner la société. Italie, France, Angleterre (XIII $-X V^{e}$ siècle), dir. Rosa-Maria Dessì, Turnhout, Brepols, 2005.

${ }^{12}$ Idees de Pau a l'Edat Mitjana, dir. Flocel Sabaté i Curull, Maite Pedrol, Lleida, Pagès, 2010.

13 Träger und Instrumentarien des Friedens im hohen und späten Mittelalter, dir. Johannes Fried, Sigmaringen, Thorbecke, 1996 ; Hermann Kamp, Friedensstifter und Vermittler im Mittelalter, Darmstadt, WBG, 2001 ; "Il n'est tresor au monde que de paix ». Hommes, lieux et instruments de pacification de l'Antiquité à nos jours, dir. Isabelle Clauzel, Boulogne-sur-Mer, Cercle d'études en Pays Boulonnais, 2007.

${ }^{14}$ Paroles de paix en temps de guerre, dir. Sylvie Caucanas, Rémy Cazals, Nicolas Offenstadt, Toulouse, Privat, 2006.

${ }^{15}$ Gerd Althoff, Spielregeln der Politik im Mittelalter. Kommunikation in Frieden und Fehde, Darmstadt, WBG, 1997; Frieden stiften. Vermittlung und Konfliktlösung vom Mittelalter bis heute, dir. Gerd Althoff, Darmstadt, WBG, 2011.

${ }^{16}$ Nicolas Offenstadt, Faire la paix au Moyen Âge. Discours et gestes de paix pendant la guerre de Cent Ans, Paris, Jacob, 2007.

${ }_{17}^{17}$ Frieden schaffen / Faire la paix, op. cit.

${ }^{18}$ Faire la guerre. Faire la paix. $136^{\mathrm{e}}$ Congrès des Sociétés historiques et scientifiques, Perpignan, 2011 ; Médiation, paix et guerre au Moyen Âge, dir. Michel Sot, édition électronique, Paris, CTHS, 2012; Faire la guerre, faire la paix: approches sémantiques et ambiguïtés terminologiques, dir. Isabelle Chave, édition électronique, CTHS, Paris, 2012; Krieg und Frieden im Übergang vom Mittelalter zur Neuzeit. Theorie - Praxis - Bilder. Guerre et paix du Moyen Âge aux temps modernes. Théories - pratiques - représentations, dir. Heinz Duchhardt, Patrice Veit, Mayence, von Zabern, 2000 ; Pace e guerra nel basso Medioevo, éd. Centro Italiano di Studi sul Basso Medioevo, Spoleto, Fondazione Centro Italiano di Studi sull'Alto Medioevo, 2004.

${ }^{19}$ Ministère des Affaires étrangères, http://basedoc.diplomatie.gouv.fr/Traites/ Accords 
peut être très difficile et nécessiter des processus de médiation, $\mathrm{d}^{\prime}$ intercession ${ }^{20}$ ou d'arbitrage. Dans ce sens, des travaux sur le règlement des conflits sont également des études sur la paix ${ }^{21}$. Le sujet est traité par des historiens, théologiens, philosophes, littéraires et des représentants d'autres disciplines scientifiques. Leurs recherches portent sur la typologie de la paix, sur certains événements marquants, ainsi que sur les traités ou négociations $^{22}$. Mais la nécessité de sauvegarder la paix et de se défendre peut également faire naître des associations ou institutions comme les Landfriedensbünde (ligues de paix territoriales) dans 1'Empire médiéval ${ }^{23}$ ou les hermandades et la Hermandad General en Espagne, dont la vocation est de sauvegarder ou d'assurer la paix ${ }^{24}$. Tout en faisant une place de choix à la quête de la paix intérieure et au rôle des femmes (comme médiatrices dans les négociations diplomatiques, comme recluses ou, dans le cas de la

Traites.php (consulté le 25 mai 2013); Heinz Duchhardt, Martin Peters, URL : http://www.ieg-mainz.de/likecms/likecms.php?site=site.htm\&dir=\&nav=85 (consulté le 25 mai 2013).

${ }^{20}$ L'intercession du Moyen Âge à l'époque moderne. Autour d'une pratique sociale, dir. Jean-Marie Moeglin, Genève, Droz, 2004.

${ }^{21}$ Le règlement des conflits au Moyen Âge, éd. Société des Historiens Médiévistes de l'Enseignement Supérieur Public (SHMESP), Paris, Publications de la Sorbonne, 2001 ; Conflitti, paci e vendette nell'Italia comunale, dir. Andrea Zorzi, Florence, Firenze University Press, 2009 ; Guerra y diplomacia en la Europa occidental 1280-1480, éd. Gobierno de Navarra, Pamplona, Fondo de Publicaciones del Gobierno de Navarra, 2005.

${ }^{22}$ Voir par exemple Arras et la diplomatie européenne (XV ${ }^{e}-X V I^{e}$ siècles), dir. Denis Clauzel, Charles Giry-Deloison, Christophe Leduc, Arras, Artois Presses Université, 1999.

23 Elmar Wadle, Landfrieden, Strafe, Recht. Zwölf Studien zum Mittelalter, Berlin, Duncker \& Humblot, 2001; Landfrieden. Anspruch und Wirklichkeit, dir. Arno Buschmann, Elmar Wadle, Paderborn, München, Schöningh, 2002 ; Horst Carl, Der Schwäbische Bund 1488-1534. Landfrieden und Genossenschaft im Übergang vom Spätmittelalter zur Reformation, Leinfelden-Echterdingen, DRW, 2000.

24 Antonio Álvarez de Morales, Las Hermandades, expresión del movimiento comunitario en España, Valladolid, 1974 ; María Asenjo González, "Ciudades y Hermandades en la Corona de Castilla. Aproximación sociopolítica », Anuario de Estudios Medievales, 27, 1997, p. 103-146. Sur la comparaison avec l'Empire médiéval voir Máximo Diago Hernando, «Die politische Rolle der Städtebünde im spätmittelalterlichen Kastilien (13. -16. Jahrhundert) », dans Frieden schaffen / Faire la paix..., op. cit., p. 139-159. 
guerre de Troie, comme pomme de discorde), les articles de ce numéro de Questes se réfèrent à plusieurs facettes du sujet.

La paix, en outre, n'est pas uniquement l'affaire des royaumes ou des princes. Il est important d'inclure des études sur le monde des villes comme celle de Laure Gevertz sur les corps de métiers londoniens. Cela vaut aussi bien pour le domaine des conflits urbains à l'échelle élargie que pour le règlement de litiges entre les membres de factions ou de métiers. Selon le constat du religieux de Saint-Denys, il ne faut pas oublier que les insurrections urbaines peuvent être «contagieuses » et, considérées comme illégitimes par les gouvernants du temps, elles peuvent mettre en danger la paix du royaume ${ }^{25}$. Dans plusieurs pays européens les villes mènent une vraie «politique étrangère ». Ainsi, dans le droit urbain de Cologne ${ }^{26}$, le droit de faire la guerre et la paix est explicitement réglé, et de grandes citésÉtats comme Venise ou des villes puissantes comme Barcelone sont des acteurs importants de la scène politique européenne. En Italie, il y eut des conflits entre les ligues urbaines et l'empereur; dans l'Empire, en 1385 et en 1449-1450, des guerres entre les princes et les villes ${ }^{27}$. Partout en Europe, la fin du XIV ${ }^{\mathrm{e}}$ siècle fut caractérisée par des troubles urbains ${ }^{28}$. Les recherches de Věra Vejrychova abordent cette période importante et traitent dans ce bulletin la paix de Tournai (1385) dans les Chroniques de Jean Froissart.

${ }^{25}$ Chronique du religieux de Saint-Denys, éd. Louis Bellaguet, Paris, Crapelet, 1842, réimpr. Paris, CTHS, 1994, t. 1, p. 130-131.

${ }^{26}$ Mandred Huiskes, «Kölns Verfassung für 400 Jahre: Der Verbundbrief vom 14. September $1396 »$, dans Quellen zur Geschichte der Stadt Köln, éd. Förderverein Geschichte in Köln, Cologne, Bachem 1996, t. 2, éd. Joachim Deeters et al., n 1, p. 6-7.

${ }^{27}$ Gisela Naegle, " "Omne regnum in se divisum desolabitur?" Coopération urbaine en France et dans l'Empire médiéval », dans Ligues urbaines et espace à la fin du Moyen Âge, dir. Laurence Buchholzer, Olivier Richard, Strasbourg, Presses universitaires de Strasbourg, 2012, p. 53-69.

${ }^{28}$ Rivolte urbane e rivolte contadine nell'Europa del Trecento: un confronto, dir. Monique Bourin, Giovanni Cherubini, Giuliano Pinto, Florence, Firenze, University Press, 2008. 
Alors que d'autres publications, en utilisant les verbes 《 rechercher », « assurer » ou « sauver », ont placé la paix dans le registre de «faire» et ont mis l'accent sur la paix comme «processus ${ }^{29}$, les auteurs de de ce numéro de Questes soulignent également le fait qu'on peut atteindre l'état de paix sans l'avoir recherché. Pour illustrer l'aspect de la quête individuelle de la paix, le choix des exemples inclut des cas extrêmes du retrait total du monde comme celui des moines du désert. Néanmoins, un tel isolement peut avoir des conséquences pour la vie de la société et sa paix : certaines formes de vie ascétique furent en effet jugées hérétiques et firent l'objet de poursuites.

Le choix des sujets est aussi une invitation au voyage dans le temps et dans l'espace. Le Livre du Chemin de Long Estude (1402) de Christine de Pizan, qui est mentionné par Sarah Delale, parle d'un voyage imaginaire : en songe, Christine et la sibylle de Cumes voyagent ensemble à travers le monde, puis dans les cieux. Les autres articles de ce recueil nous mènent des recluses d'Amiens étudiées par Julie Pilorget, de la paix intérieure et de la solitude à l'activité et aux conflits des métiers de Londres. Le rôle des femmes comme médiatrices de paix dans la principauté latine de Morée en Grèce byzantine est commenté du point de vue des auteurs médiévaux français, grecs et aragonais par Marie Guérin. Dans l'Historia destructionis Troiae, présentée par Marie Bedel, le sicilien Guido delle Colonne, traite en latin un sujet d'origine grecque qui était également connu par des œuvres comme le Roman de Troie « en romanz ». Ainsi, la paix pose-t-elle également la question des relations interculturelles.

${ }^{29}$ Pierre Monnet, "Conclusions », dans Frieden schaffen / Faire la paix..., op. cit., p. 357-372, ici p. 358-359 ; Jean-Marie Moeglin, « À la recherche de la "paix finale". Guerre et paix dans les relations des rois de France et d'Angleterre au XIV ${ }^{\mathrm{e}}$ siècle: références normatives et pratiques politiques », dans ibid., p. 51-82. 